\title{
Directed growth of hydrogen lines on graphene: High-throughput simulations powered by evolutionary algorithm
}

\author{
Özbal, G.; Falkenberg, J.T.; Brandbyge, M.; Senger, R. T.; Sevincli, H.
}

Published in:

Physical Review Materials

Link to article, DOI:

10.1103/PhysRevMaterials.2.073406

Publication date:

2018

Document Version

Publisher's PDF, also known as Version of record

Link back to DTU Orbit

Citation (APA):

Özbal, G., Falkenberg, J. T., Brandbyge, M., Senger, R. T., \& Sevincli, H. (2018). Directed growth of hydrogen lines on graphene: High-throughput simulations powered by evolutionary algorithm. Physical Review Materials, 2(7), [073406]. https://doi.org/10.1103/PhysRevMaterials.2.073406

\section{General rights}

Copyright and moral rights for the publications made accessible in the public portal are retained by the authors and/or other copyright owners and it is a condition of accessing publications that users recognise and abide by the legal requirements associated with these rights.

- Users may download and print one copy of any publication from the public portal for the purpose of private study or research.

- You may not further distribute the material or use it for any profit-making activity or commercial gain

- You may freely distribute the URL identifying the publication in the public portal 


\title{
Directed growth of hydrogen lines on graphene: High-throughput simulations powered by evolutionary algorithm
}

\author{
G. Özbal, ${ }^{1}$ J. T. Falkenberg, ${ }^{2,3}$ M. Brandbyge, ${ }^{2,3}$ R. T. Senger, ${ }^{1}$ and H. Sevinçli ${ }^{4, *}$ \\ ${ }^{1}$ Physics Department, Izmir Institute of Technology, 35430 Izmir, Turkey \\ ${ }^{2}$ Department of Micro- and Nano-technology, Technical University of Denmark, DK-2800 Kongens Lyngby, Denmark \\ ${ }^{3}$ Center for Nanostructured Graphene (CNG), Department of Micro- and Nano-technology, Technical University of Denmark, \\ DK-2800 Kongens Lyngby, Denmark \\ ${ }^{4}$ Department of Materials Science and Engineering, Izmir Institute of Technology, 35430 Izmir, Turkey
}

(Received 22 April 2018; published 31 July 2018)

\begin{abstract}
We set up an evolutionary algorithm combined with density functional tight-binding calculations to investigate hydrogen adsorption on flat graphene and graphene monolayers curved over substrate steps. During the evolution, candidates for the new generations are created by adsorption of an additional hydrogen atom to the stable configurations of the previous generation, where a mutation mechanism is also incorporated. Afterwards a two-stage selection procedure is employed. Selected candidates act as the parents of the next generation. The evolutionary algorithm predicts formation of lines of hydrogen atoms on flat graphene. In curved graphene, the evolution follows a similar path except for a new mechanism, which aligns hydrogen atoms on the line of minimum curvature. The mechanism is due to the increased chemical reactivity of graphene along the minimum radius of curvature line (MRCL) and to $\mathrm{sp}^{3}$ bond angles being commensurate with the kinked geometry of hydrogenated graphene at the substrate edge. As a result, the reaction barrier is reduced considerably along the MRCL and hydrogenation continues like a mechanical chain reaction. This growth mechanism enables lines of hydrogen atoms along the MRCL, which has the potential to overcome substrate or rippling effects and could make it possible to define edges or nanoribbons without actually cutting the material.
\end{abstract}

DOI: 10.1103/PhysRevMaterials.2.073406

One of the most attractive features of two-dimensional materials is their ability to be functionalized in more effective ways than conventional materials. However, graphene lacks an energy band gap, a requirement for graphene to be useful in digital electronics applications. Adsorption of hydrogen atoms is a direct way to alter chemical and physical properties of graphene [1] and hydrogenation in a controlled way was shown to open a band gap [2-9]. There are proposals to tune the band gap of graphene by hydrogen adsorbed nanoripples [1012] and forming superlattices consisting of lines of adsorbed hydrogen atoms [13,14]. It is well known that curvature enhances chemical reactivity of graphene and related materials by decreasing the activation barrier for adsorbates [15-22]. This property was used to enhance the hydrogen storage capacity of graphene and CNTs [23-27]. A straightforward way to deform graphene is to introduce steps in the underlying substrate. Such deformation also affects electronic and thermal transport properties [28-30].

Stability of small hydrogen clusters on single and multilayer graphene, as well as on carbon nanotubes, has been studied by several groups theoretically and experimentally [17,31-40]. Also there have been attempts to determine the most stable graphane like clusters and to control the size of the hydrogen islands $[41,42]$. Those studies were focused either on individual dimer and trimer configurations [32,36] or the configurations were generated from extensions of ortho- and parapositions

\footnotetext{
*haldunsevincli@iyte.edu.tr
}

[31], but progression of the hydrogenation process was not addressed on flat or curved graphene.

Here, we perform high-throughput calculations within an evolutionary framework. We demonstrate formation of lines of hydrogen atoms on flat and curved graphene sheets from one side. On curved graphene, hydrogenation is predicted to take place as a chain reaction and long lines of hydrogen atoms are shown to be energetically more favorable along the minimum radius of curvature line (Fig. 1).

Methods. We employ a density functional theory based tight-binding (DFTB) method, where we use the DFTB+ code [43] and the mio-1-1 parameter set [44]. Density functional theory (DFT) is also used to check the compatibility of the two methods $[45,46]$. Periodic boundary conditions are employed in all simulations. For flat graphene, a $7 \times 7 \times 1$ supercell of the primitive unit cell is used (see Fig. 2) with a $k$-point sampling of $10 \times 10 \times 1$ in the Monkhorst-Pack scheme [47]. We fix the supercell lattice parameters corresponding to the carbon-carbon distance of pristine graphene. The vacuum region is set to $10 \AA$ to avoid interplane interactions for flat geometry, while it is chosen to be larger than $50 \AA$ for the curved geometries. A unit cell which consists of 144 atoms is built for obtaining the curved geometry and $1 \times 10 \times 1$ supercells of this unit cell are used (see Fig. 2) with $k$-point samplings of $1 \times 3 \times 1$ or higher. Structures are optimized until the maximum force component is below the $2 \times 10^{-2} \mathrm{eV} / \AA$. We have checked the strain on graphene supercell after adsorption of hydrogen atoms by reoptimizing the lattice parameters. We have used the most stable hexamer configuration and found that the supercell 


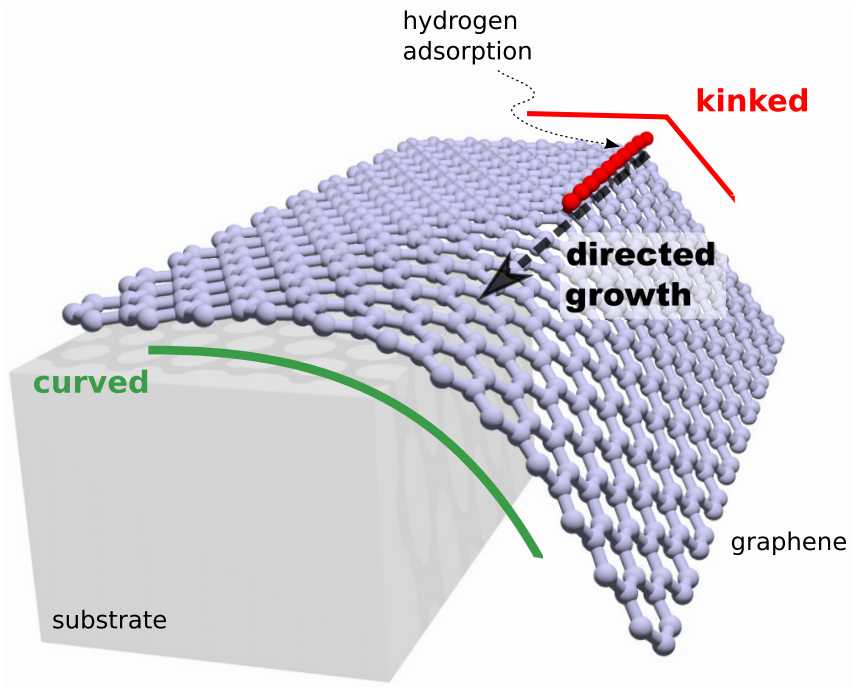

FIG. 1. Illustration of the directed growth of hydrogen atoms on the minimum radius of curvature line. Propagation of the hydrogen atoms along the first and the second minimum radius of curvature lines leads to formation of a kink line.

has shrunk by only $0.35 \%$, which indicates that the adsorption induced strain does not affect our simulations considerably. We disregard spin polarization in our calculations since the energy difference due to spin polarization is much less than the threshold in the evolutionary algorithm as explained below.

Flat graphene. There are several previous studies related to structural properties of hydrogen adsorption on flat graphene [31-38]. It was shown that the most preferable positions for hydrogen dimers are the ortho- and parapositions. Ortho (O)-, meta $(\mathrm{M})$-, and para $(\mathrm{P})$-positions correspond to the first, second, and third nearest neighboring sites, respectively (see Fig. 2). Trimer and tetramer adsorption follows extensions of ortho- and parapositions consequently. Before performing high-throughput DFTB simulations, we compare DFTB results against DFT for a relatively large set consisting of 60 configurations, which cover the configurations investigated in the literature $[31,32,36]$. Details of the comparison are given in the Supplemental Material (Fig. S14) [48], which clearly show that DFT and DFTB results are in very good agreement.
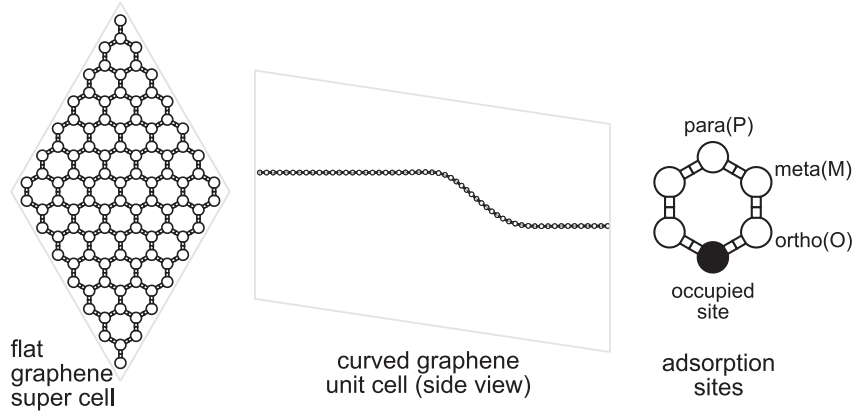

FIG. 2. Flat and curved graphene structures are shown and the adsorption sites are labeled as ortho (O)-, para (P)-, and meta (M)configurations with respect to the occupied site.

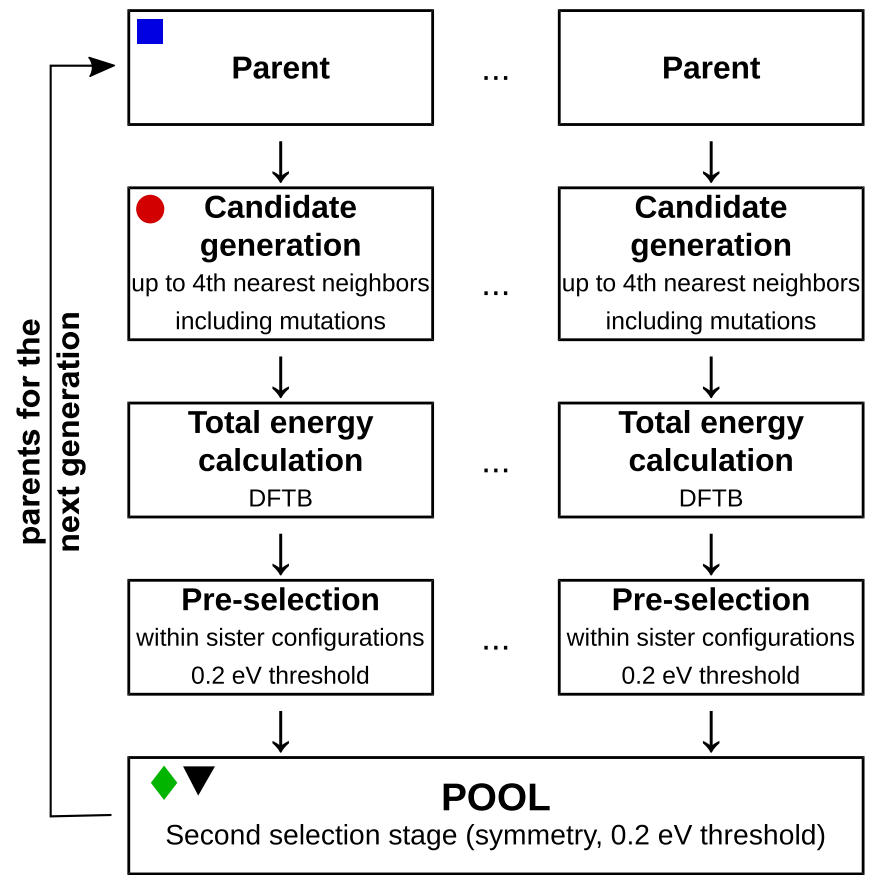

FIG. 3. Flow chart of the evolutionary algorithm. The algorithm starts with generation of candidate configurations (red circles) from the parent configurations (blue squares). After DFTB based total energy calculation of the relaxed geometries, preselection is performed among the sister configurations, where the threshold energy is set to $0.2 \mathrm{eV}$. Preselected configurations are collected at a pool and a second selection procedure is carried out taking total energy and symmetry properties into consideration. Successful candidates (green diamonds) are considered as the stable configurations of the current generation and they are used as parents for the next generation. Candidates that are eliminated at the pool-selection stage are marked with black triangles. (Blue squares, green diamonds, and black triangles are used correspondingly in Figs. 4, 6, and 7, whereas red circles are used in Figs. S2-S8 [48].)

We study hydrogen adsorption on flat graphene by using an evolutionary approach, which relies on the selection of favorable configurations by comparing their total energies (see Fig. 3). The algorithm starts with a monomer as the parent configuration, from which the candidate dimer configurations are generated. The candidate generations include all available adsorption sites up to the fourth nearest neighboring site of the existing occupied sites. The total energies of the relaxed candidate configurations are calculated. The threshold value for the preselection stage is set to $0.2 \mathrm{eV}$ above the minimum energy of the members of the same family, i.e., candidates originating from the same parent. Afterwards, a second selection stage is performed, which will be referred to as the pool selection. In pool selection, the energy threshold is set to $0.2 \mathrm{eV}$ above the global minimum energy of all candidates in the pool. Binding of a hydrogen atom induces an out-of-plane displacement of the carbon atom, which leads to an activation barrier of $0.2 \mathrm{eV}$. According to previous studies, chemisorption can take place if hydrogens surpass this activation barrier [31,35,38,38,49-51]. For this reason, $0.2 \mathrm{eV}$ is chosen as a threshold value. Symmetry is the second criterion during the pool selection. If there are geometrically equivalent 
candidates due to symmetries, only one of them is proceeded to the next generation. The successful candidates, i.e., stable configurations, are then the parents for the next generation.

Starting from dimers, we include a mutation mechanism during candidate generation. Mutations alter the configurations of the parent by hopping one of the hydrogens to an available nearest neighboring site. Afterwards, the candidates are generated by adsorption of a hydrogen atom to up to the fourth nearest neighboring site, as usual. We simulated all possible mutations on flat graphene from dimers to hexamers, which make 5355 configurations in total. The predicted stable configurations up to tetramers are in exact agreement with literature. Above this size, the evolutionary algorithm finds stable configurations which were not predicted before.

The results of the high-throughput simulations employing the evolution scheme are summarized in Fig. 4. Different generations are grouped as rows and depicted with dashed lines. For each generation the number of panels is the sum of the number of successful candidates from previous generation and the number of mutants that could yield stable configurations. The parents are marked with blue squares, while the green diamonds show the members of the new generation, i.e., the candidates which succeed both selection stages. All candidate configurations are shown in Figs. S2-S6 [48] with red circles. If the selection process was carried out only within sister configurations (i.e., without pool selection) the new generation would have a higher number of stable configurations. The configurations that are eliminated during pool selection are marked with black triangles.

In the first row of Fig. 4, one observes that only two candidates, $\mathrm{D}_{2}$ and $\mathrm{D}_{4}$, are selected for the new generation from the parent monomer. They correspond to the orthoand parapositions. These configurations are selected from 72 candidates (see Fig. S2 [48]), which are composed of one nonmutant and three mutant parents. In fact, there are six favorable adsorption sites but four members $\mathrm{D}_{3}, \mathrm{D}_{5} ; \mathrm{D}_{1}, \mathrm{D}_{6}$ are equivalents of $D_{2}$ and $D_{4}$, respectively. In agreement with the previous results, the metaposition is found to be unfavorable. The orthopositioned configuration is more stable than the parapositioned configuration, and there is only $59.2 \mathrm{meV}$ energy difference between them. There is no direct correlation between the dihedral angles and the binding energies, but the C-H bond lengths (1.129 ̊, $1.138 \AA$, and $1.142 \AA$ for ortho, para, and meta, respectively) follow the same trend with the binding energies.

In generating trimers from dimers, there exist two nonmutant and 10 mutant parents, which enable 298 candidates (see Fig. S3 [48]). At the preselection stage, five successful configurations $\left(\operatorname{Tr}_{7}, \mathrm{Tr}_{8}, \mathrm{Tr}_{9}, \mathrm{Tr}_{10}\right.$, and $\left.\operatorname{Tr}_{11}\right)$ are found from two parents $\left(\mathrm{D}_{2}\right.$ and $\left.\mathrm{D}_{4}\right)$, one being a mutant $\left(\operatorname{Tr}_{9}\right)$ (see the third panel in the second row in Fig. 4). The mutation switches a para configuration to a meta configuration, which is known to be unstable. It is then stabilized by adsorption of a hydrogen atom to the common orthopositions of both parent atoms. The stabilization mechanism is in agreement with the literature in the sense that the metaposition is not favorable, while the orthoposition is. Two configurations $\left(\operatorname{Tr}_{10}\right.$ and $\left.\operatorname{Tr}_{11}\right)$ are eliminated due to symmetry. The stable configuration $\operatorname{Tr}_{8}$ occupies $\mathrm{P}-\mathrm{O}$ positions, whereas $\mathrm{Tr}_{7}$ occupies P-P positions.
For tetramers there exist three nonmutant and 21 mutant parents which generate 737 candidates (see Fig. S4 [48]). Among those candidates, we find four stable configurations $\left(\mathrm{Te}_{14}, \mathrm{Te}_{17}\right.$, $\mathrm{Te}_{18}$, and $\left.\mathrm{Te}_{19}\right)$ from three parents $\left(\operatorname{Tr}_{7}, \operatorname{Tr}_{8}\right.$, and $\left.\operatorname{Tr}_{9}\right)$, one being a mutant $\left(\mathrm{Te}_{14}\right)$ which can be seen in the third row of Fig. 4. The mutation alters a $\mathrm{P}-\mathrm{P}$ configuration to a $\mathrm{P}-\mathrm{M}$ configuration and with the new hydrogen we obtain a P-O-O geometry. It is again confirmed that metaposition is unfavorable. In the tetramer family, five members failed to continue to the next generation at the pool-selection stage (indicated with black triangles). Configuration $\mathrm{Te}_{17}$ is composed of $\mathrm{P}-\mathrm{O}-\mathrm{O}$ sites, whereas $\mathrm{Te}_{18}$ displays P-O-P geometry. One can make some predictions from the obtained results already. $\mathrm{Te}_{17}$ and $\mathrm{Te}_{18}$ are precursors of linear chains, whereas a deviation from the linear geometry appears in $\mathrm{Te}_{14}$. The evolution of $\mathrm{Te}_{19}$ is rather indeterminate. It can either form a hexagonal ring or evolve into a double chain with armchair-type geometry. The former indicates clustering, while the latter stands for linear patterns.

Next, we generate pentamers from tetramers. There exist four nonmutant and 32 mutant parents, which generate 1242 candidates (see Fig. S5 [48]). Fourth row of Fig. 4 shows that the new generation is found to have seven stable members $\left(\mathrm{P}_{21}\right.$, $\mathrm{P}_{22}, \mathrm{P}_{23}, \mathrm{P}_{26}, \mathrm{P}_{28}, \mathrm{P}_{29}$, and $\left.\mathrm{P}_{30}\right)$ from four parents $\left(\mathrm{Te}_{14}, \mathrm{Te}_{17}\right.$, $\mathrm{Te}_{18}$, and $\mathrm{Te}_{19}$ ). The new generation of pentamers does not involve new mutations. All members of the new generation are composed of either only orthopositioned or mixtures of ortho- and parapositioned hydrogens. There are no O-O-O$\mathrm{O}$ configurations; therefore, formation of a hexagonal ring is energetically suppressed at this stage. More interestingly, lines with armchair-type geometry become the most favorable configurations.

Hexamer configurations are the last step for our calculations on flat graphene. When we consider the results of the hexamer generations, there exist seven nonmutant and 69 mutant parents which produce 3006 candidates (see Fig. S6 [48]). From those candidates, the new generation contains 10 nonmutant stable members $\left(\mathrm{H}_{37}, \mathrm{H}_{44}, \mathrm{H}_{45}, \mathrm{H}_{46}, \mathrm{H}_{47}, \mathrm{H}_{48}, \mathrm{H}_{50}, \mathrm{H}_{52}, \mathrm{H}_{53}\right.$, and $\mathrm{H}_{54}$ ), which can be seen in the fifth and sixth rows of Fig. 4. We eliminate 13 of the candidates due to symmetry or because of the ground state coming from a nephew, namely at the poolselection stage. $\mathrm{H}_{37}$ is a mixture of linear and armchair-type geometries, resembling a broken line. $\mathrm{H}_{38}$ is eliminated due to the presence of an equivalent, $\mathrm{H}_{52}$.

We have checked the strain on graphene supercell after adsorption of six hydrogen atoms by reoptimizing the lattice parameters for the armchair configuration. We find that the supercell has shrunk by only $0.35 \%$, which indicates that the adsorption induced strain does not affect our simulations considerably.

In summary, we generated a pool which is composed of a total of 5355 candidates from dimers to hexamers. It is clear that ortho- and para extended combinations are favored, whereas combinations which consist of metaposition are not. The most striking and remarkable result is the alignment of hydrogen atoms in armchair-type geometry and $\mathrm{H}_{53}$ is found to be energetically the most preferable configuration. The next most favorable configuration is $\mathrm{H}_{45}$, which has a linear alignment with hydrogen atoms occupying $A$ and $B$ sublattices evenly. An important note is in order here. Both armchairtype geometry and single line formation are in the armchair 
D

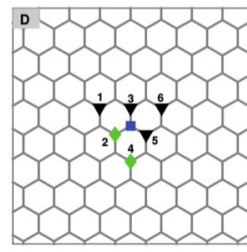

$\operatorname{Tr}$

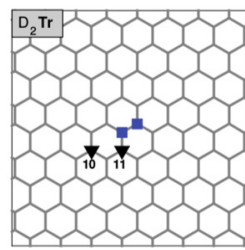

Te

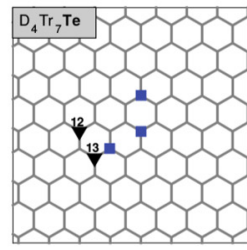

H
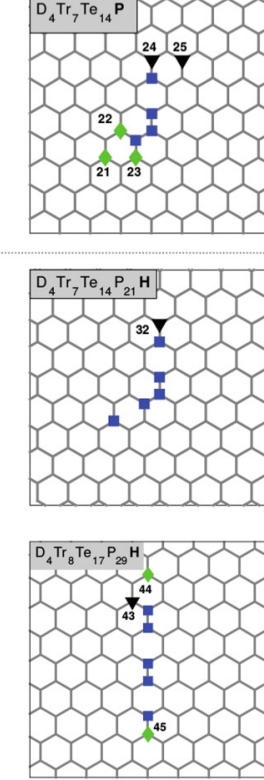
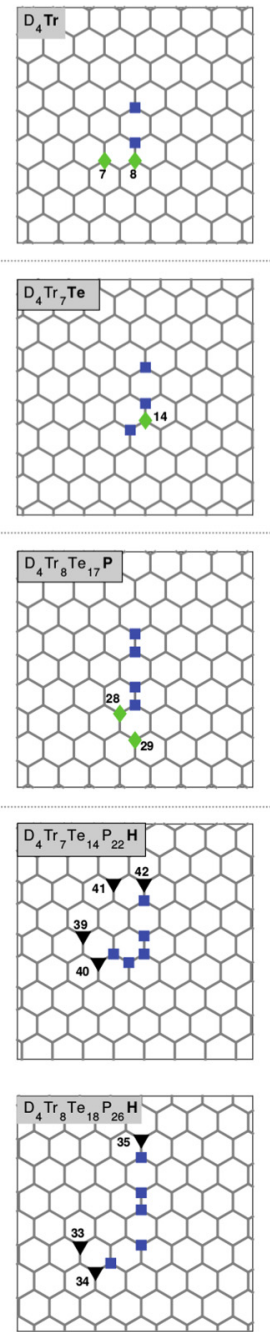
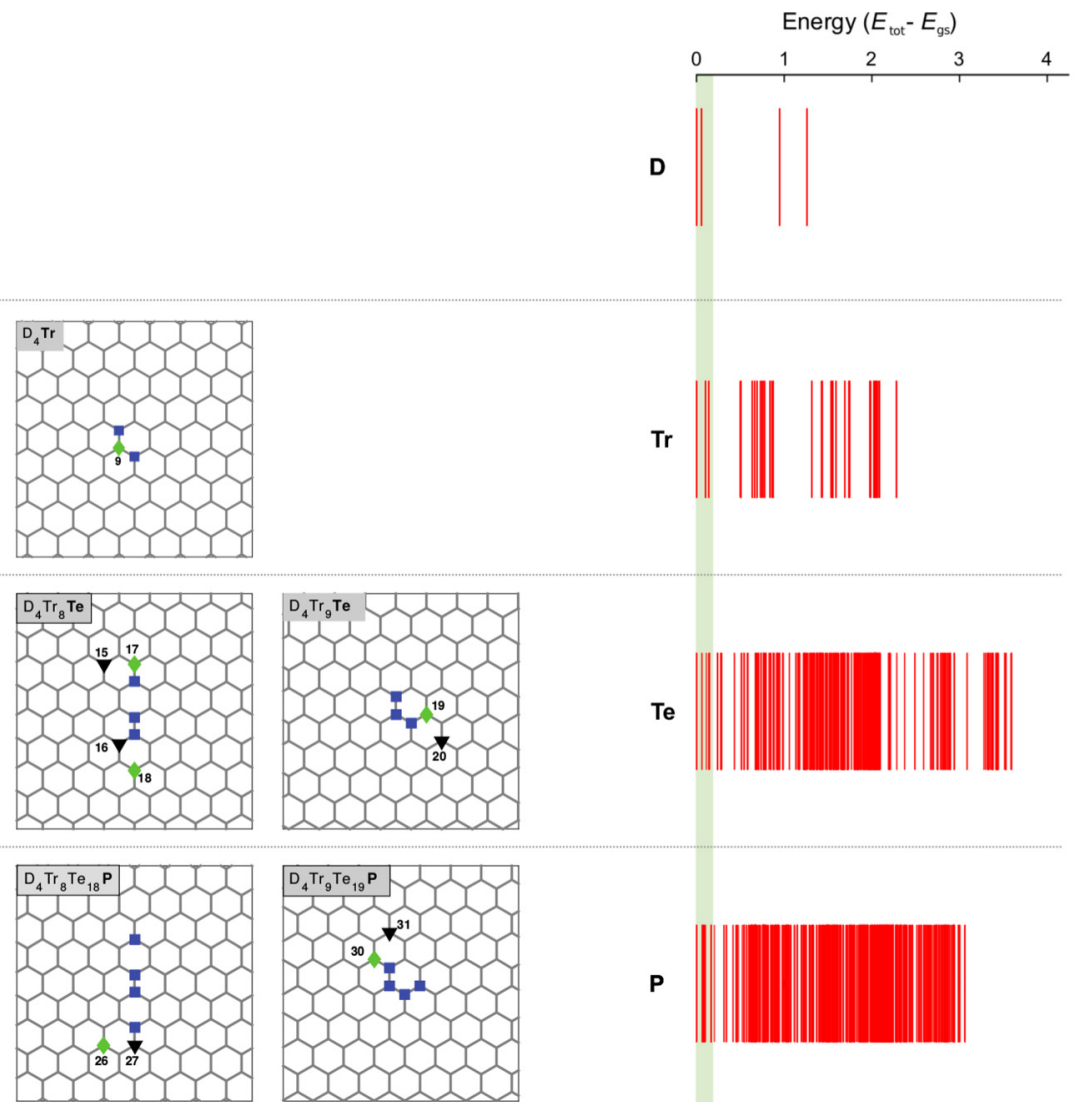

Te
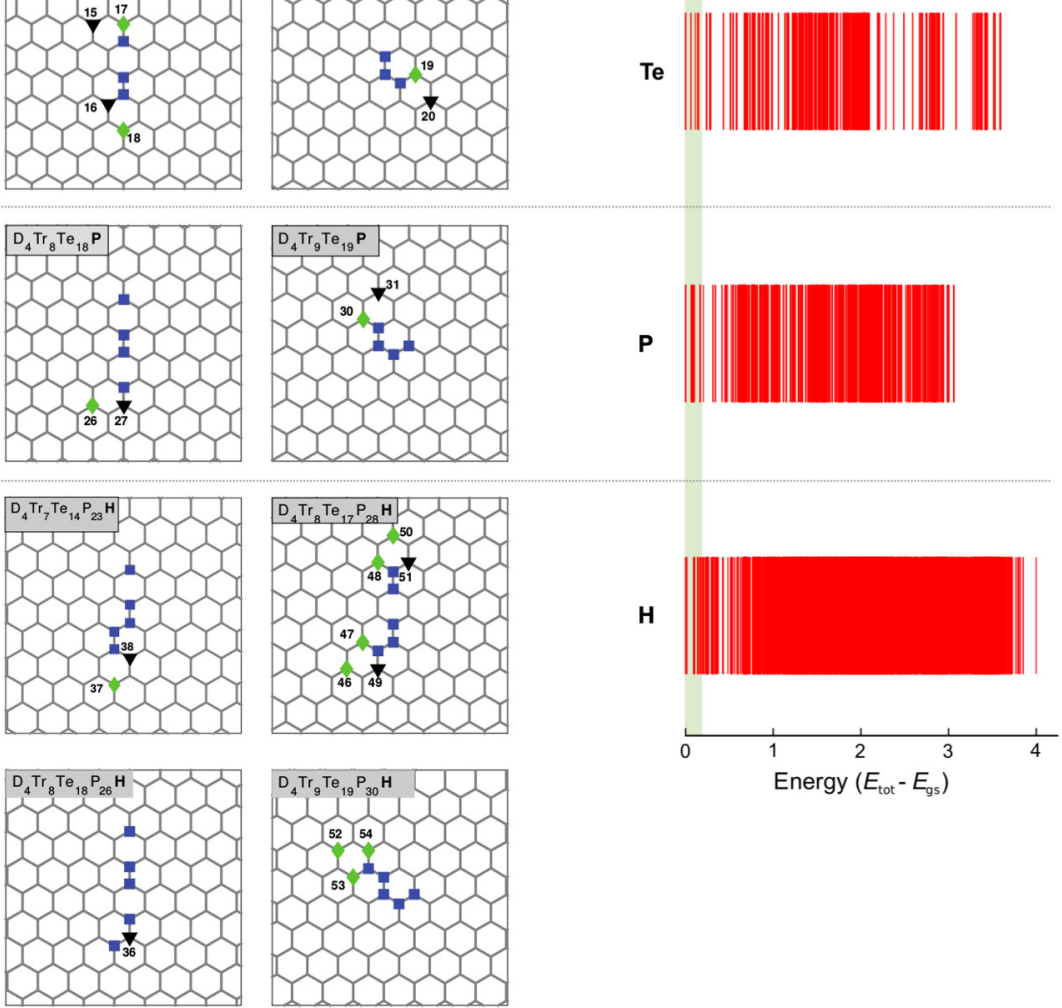

D $1{ }^{2}{ }^{2} \quad 3$

$\operatorname{Tr} \quad 1011$
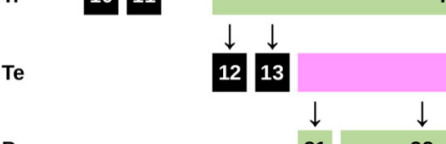

$\underline{14}$

P

$\underline{21} \quad \underline{22} \quad \underline{23} \underline{24} \underline{25}$
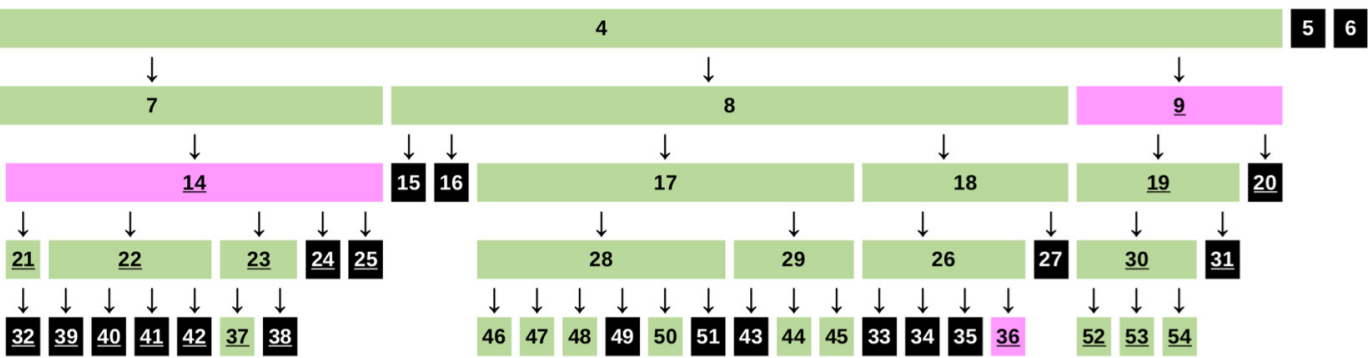

FIG. 4. Evolution of generations on flat graphene. Parent atoms (blue squares), stable configurations (green diamonds), and configurations that are eliminated at the pool selection stage (black triangles) are shown throughout the evolution (upper left). Generations are separated with dotted lines and denoted as $\mathrm{D}, \mathrm{Tr}, \mathrm{Te}, \mathrm{P}$, and $\mathrm{H}$ for dimers, trimers, tetramers, pentamers, and hexamers, respectively. Each panel corresponds to a family; the family tree is denoted at the upper left corner of each panel. The evolution of generations can also be tracked at the lower panel, where each row stands for a generation and each box for a candidate with the same enumeration with the geometry plots. The stable, eliminated, and mutant configurations are shown with green, black, and pink boxes, respectively. Relative total energies of simulated configurations are shown on the right, where the green zone indicates the $200 \mathrm{meV}$ threshold value. The complete list of simulated geometries can be found in Figs. S2-S6 and their corresponding energies at Table SI [48]. See also the Supplemental Material [56]. 

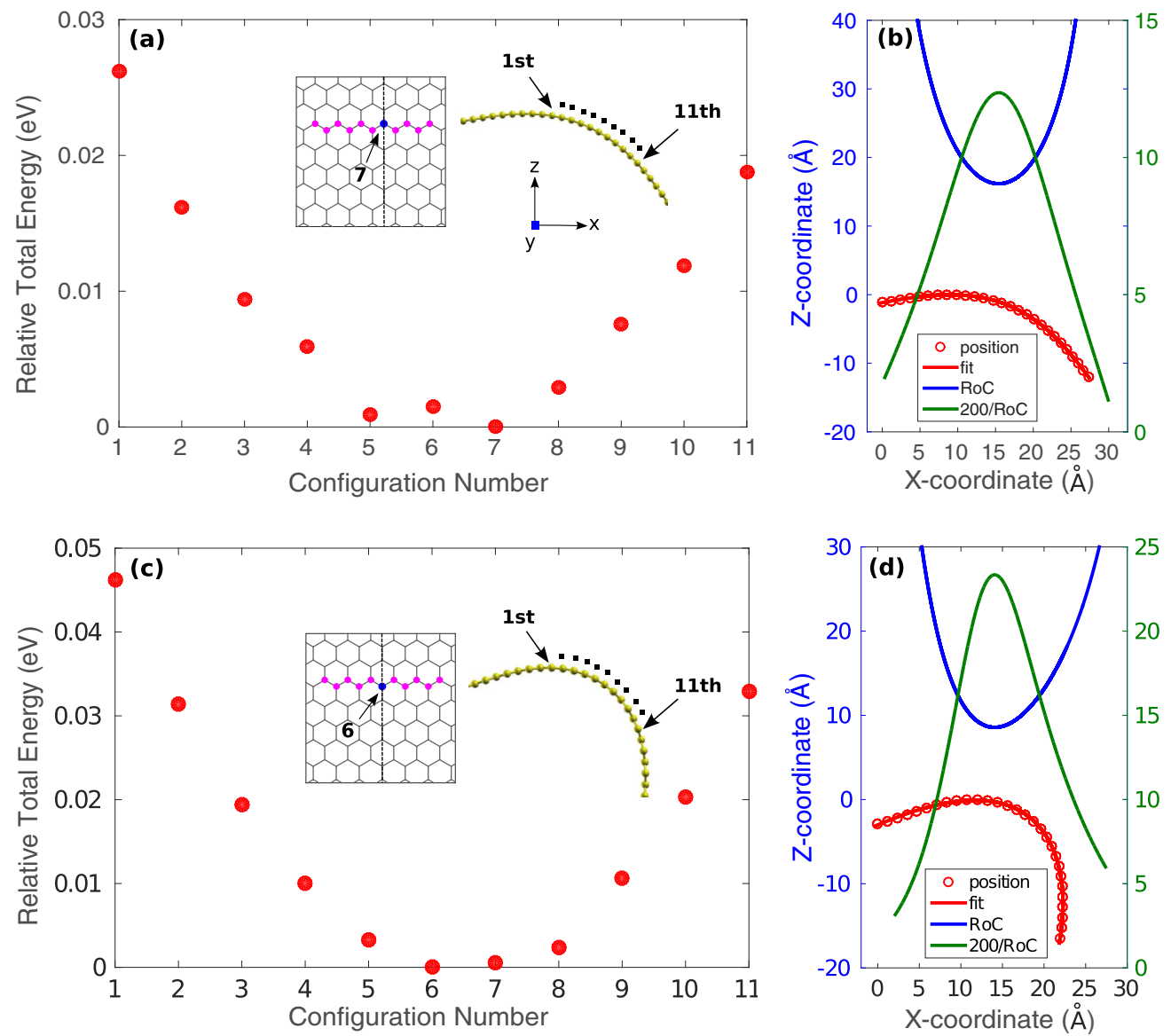

FIG. 5. Curvature and binding to curved graphene. Energetics of single hydrogen adsorption on curved graphene with bending angles $52^{\circ}$ and $90^{\circ}$ [(a) and (c), respectively]. In the insets top view and side view of the configurations are illustrated. Pink dots denote tested configurations and blue dots show most favorable positions. Configurations 7 and 6 ensure the positions of the minimum radius of curvature lines (MRCL) for $52^{\circ}$ and $90^{\circ}$ bending. The MRCLs are depicted in the insets. In (b) and (d) red circles indicate graphene atoms, the blue lines show the radius of curvature (ROC), and the green lines show the inverse radius of curvature (IROC).

direction, the former being a double line and consisting of orthopositions only. In addition, $\mathrm{H}_{44}$ is less stable than $\mathrm{H}_{45}$ because of unpaired sublattice sites. Energy difference between $\mathrm{H}_{45}$ and $\mathrm{H}_{53}$ is only $2.67 \mathrm{meV}$ per adsorbant. The sixfold para configuration (see Conf-2 in Fig. S13 [48]) is a special case, whose relative total energy is lower than line formations. It does not appear as a stable configuration at the end of the evolution procedure, because its precursor was eliminated at the pool selection stage of tetramers.

Armchair-type line formation was previously shown to be more stable than zigzag-type line formation, as well as formation of triangular and circular clusters [52], but single lines were not investigated in that work. Single line geometries of tetramers were reported on bilayer graphene but longer lines were not observed [53]. On graphene/SiC(0001) line formations were reported to be as short as a dimer [54], which are in either ortho- or para configurations. Interestingly, scanning tunneling microscopy images show important electronic contribution of the substrate and the modulation in adsorption energy was reported to be as high as $230 \mathrm{meV}$. Similarly, Moiré superstructures are known to influence hydrogen adsorption on graphene [2]. Therefore, we speculate that the lack of experimental observation of hydrogen lines on flat graphene may be due to the substrate effects.
Curved graphene. A strategy to overcome these effects could be incorporating bending so that substrate induced ripples are overridden by a strong bending and the potential landscape altered by the substrate becomes a minor ingredient compared to the increased chemical reactivity along the minimum radius of curvature line (MRCL). In what follows, we examine hydrogen line formation on curved graphene surfaces.

Armchair direction has the lowest bending modulus [55] and it is also the favored direction for formation of hydrogen lines. Therefore, we consider the substrate direction to be aligned with the armchair direction. Periodic boundary conditions in both directions, parallel and perpendicular to the step edge, are applied. Instead of simulating the atomistic details of the substrate, its influence is included by bending graphene over a step. However, long range interactions like hydrogen substrate interaction are neglected. Bending is achieved through constrained relaxation of the atomic positions, where the atoms away from the step edge are fixed and those close to the step edge are free during optimization of the atomic forces. The bending angles are chosen to be $52^{\circ}$ and $90^{\circ}$, which are determined by considering the bond angles and the strength of substrate graphene interaction. The $52^{\circ}$ corresponds to the projection of the tetrahedral angle of $\mathrm{sp}^{3}$ hybridized carbons, and $90^{\circ}$ stands for strong interaction between graphene and 

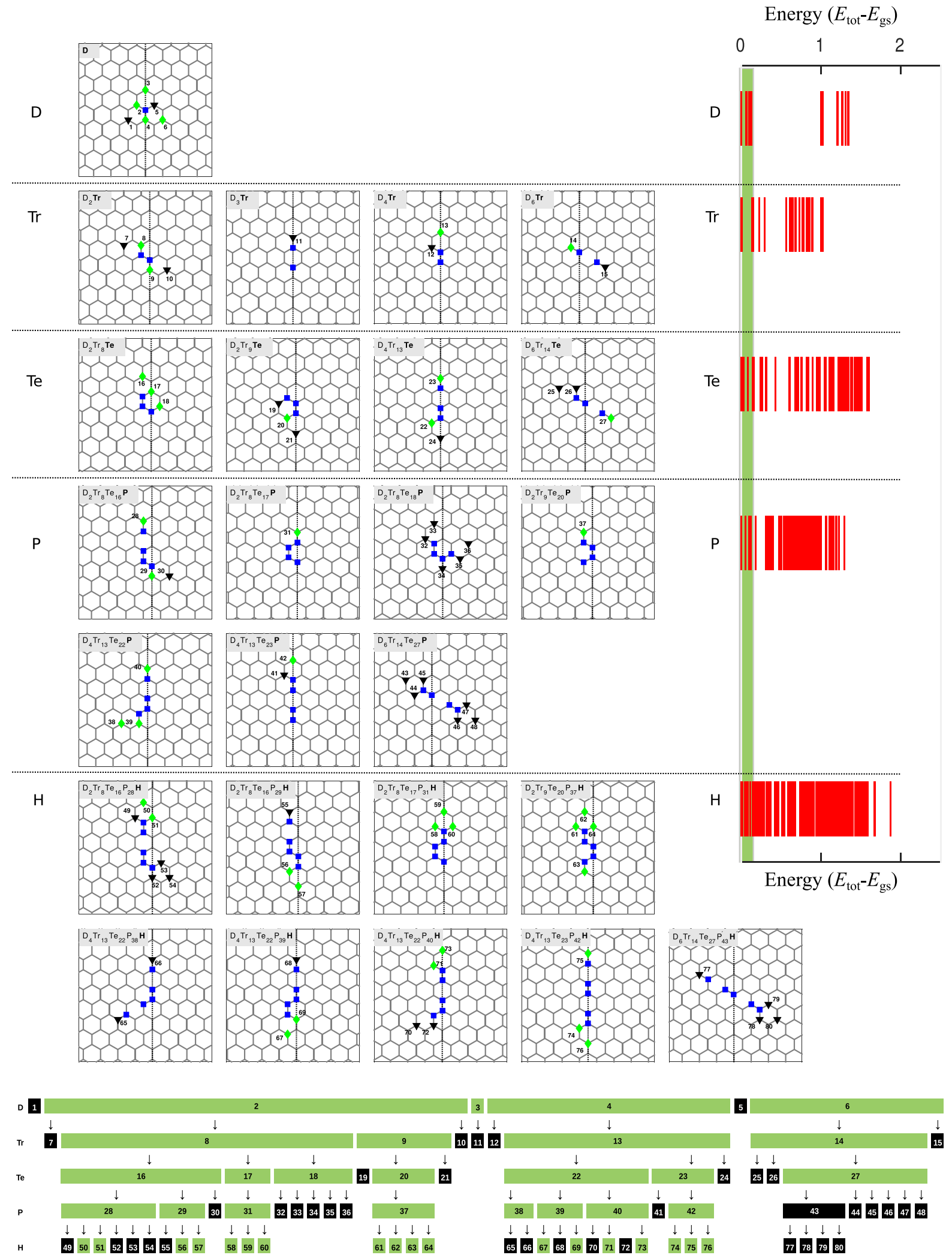

FIG. 6. Evolution of generations during the hydrogenation of curved graphene $\left(52^{\circ}\right)$. The same color codes and notation are applied as in Fig. 4. The complete list simulated structures can be found in Fig. S7 [48].

the substrate. In the absence of hydrogenation, the bending angles are determined solely by the interaction strength. The radius of curvature (ROC) is defined as $R=\left|\left(1+y^{\prime}\right)^{3 / 2} / y^{\prime \prime}\right|$ and calculated numerically using the interpolated curve. The lengths of the curved parts across the step are chosen so as to remove the tensile stress after hydrogenation. For different interaction strengths, which correspond to different substrates, ROC is found to be in the range between $13.5 \AA$ and $3.3 \AA$, 
where the step height is kept as $10 \AA[28,30]$. In Figs. 5(b) and 5(d) the positions of carbon atoms (red circles) and the fitted curve (red curve) are plotted together with the calculated ROC (blue) and its inverse (green). In Figs. 5(a) and 5(c), the total energies are plotted with reference to the minimum energy configurations as the adsorption site is varied for both bending angles. Top and side views of tested adsorption sites are indicated from 1 to 11 in the insets. The reactivity of graphene increases with reduced ROC, and the total energy increases almost symmetrically with increasing ROC, where the minimum energy is achieved at the site with the smallest ROC. In $52^{\circ}$ bending the minimum ROC is $16 \AA$, while in $90^{\circ}$ bending we find the minimum ROC close to $10 \AA$, which are close to the previous results [30]. We also perform single hydrogen adsorption calculations on curved graphene for $90^{\circ}$ bending by using SIESTA. All trends in total energy from DFT are reproduced by DFTB (see Fig. S14 [48] and Fig. S15 [48]). Binding energies are calculated using

$$
E_{\text {binding }}^{(n)}=\left(E_{\text {graphene }}+n E_{\mathrm{H}}-E_{\text {graphene }+\mathrm{H}_{\mathrm{n}}}\right) / n .
$$

The binding energies of single hydrogen on $90^{\circ}$-bent graphene are larger than those of the $52^{\circ}$-bent graphene due to the curvature effect (see Table S-XIII [48] and Table S-XVI [48] for $52^{\circ}$ bending and Table S-XVIII [48] and Table S-XXI [48] for $90^{\circ}$ bending).

The number of atoms in the simulation cell is significantly larger for curved graphene. On top of that, the number of possible configurations is multiplied because of the broken symmetries due to bending. These make it impossible to simulate all possible configurations. Therefore, equipped with the information from the evolution on flat graphene, we reduce the number of candidates significantly on curved graphene by focusing on the formation of lines of hydrogen atoms.

$52^{\circ}$ bending. In Fig. 5(a), it is shown that configuration7 is the most favorable adsorption site for hydrogen on curved graphene $\left(52^{\circ}\right)$, which coincides with the MRCL. Configuration-7 is taken as the parent for the dimer generation. Successful candidates as well as those eliminated during the pool selection are shown in Fig. 6, while all tested candidates on $52^{\circ}$-bent graphene are presented in Fig. S7 [48]. We note that enumeration of configurations in Fig. S7 [48] is independent of the enumeration in Fig. 6.

For dimers, we consider 21 candidates, which are distributed equally on the left- and the right-hand sides of the MRCL (see first row of Fig. S7 [48]). At the preselection stage, six candidates $\left(\mathrm{D}_{1} \ldots \mathrm{D}_{6}\right)$ are found to be stable (Fig. 6). When the selection process is considered, dimers $\left(52^{\circ}\right)$ produced more parents for the next generation than flat graphene $\left(0^{\circ}\right)$ due to the symmetry breaking with the curvature. However, these parents are ortho- and parapositioned hydrogens as in flat graphene. Only two candidates $\mathrm{D}_{1}$ and $\mathrm{D}_{5}$ are eliminated during the pool selection. Relative total energies and binding energies per hydrogen atom of 21 candidates are summarized in Table S-II [48] and Table S-XII [48], respectively. The most favorable dimer configuration is found to be $\mathrm{D}_{4}$ on $52^{\circ}$-bent graphene.

For trimers, 43 candidates are generated from four parents (see the second row of Fig. S7 [48]). Only four of them succeed after the selection process (see Fig. 6). For all trimer configura- tions considered, $\mathrm{P}-\mathrm{O}$ positioned $\operatorname{Tr}_{11}$ and $\mathrm{P}-\mathrm{O}$ positioned $\operatorname{Tr}_{14}$ making a $60^{\circ}$ angle with the MRCL are found to be the most stable configurations. It is interesting that no P-P positioned configurations appear in $52^{\circ}$ bending, whereas P-P positioned $\mathrm{D}_{4} \operatorname{Tr}_{7}\left(0^{\circ}\right)$ is one of the most favorable configurations. It is expected that a P-P positioned configuration would be generated from $\mathrm{D}_{3} \operatorname{Tr}\left(52^{\circ}\right)$ but two of the parent's atoms are located on the MRCL and this causes an increase in the energy cost to adsorb a third hydrogen at the paraposition. Relative total energies and binding energies per hydrogen are summarized in Table S-III [48] and Table S-XIII [48], respectively.

Tetramer generation consists of 94 candidates from four parents (see the third row of Fig. S7 [48]). Seven candidates are selected as parents for the next generation. Tetramer configurations that are stable on flat graphene are favored on the $52^{\circ}$-bent case, as well (Fig. 6). One should note that the stable configurations are either mixtures of orthoand parapositions or pure orthopositioned configurations. In addition, pure P-P-P positioned configurations are eliminated at the preselection stage in $52^{\circ}$ bending, while those of flat graphene are eliminated at pool-selection stage. Another point is O-O-P positioned $\mathrm{Te}_{16}$ tends to form a single line on the second MRCL, the parallel line on the left or on the right of the MRCL. In family $\mathrm{D}_{2} \mathrm{Tr}_{9} \mathrm{Te}, \mathrm{Te}_{20}$ is kept in order to observe the effect of a shift in the position parallel to the MRCL in the subsequent generation. It can be seen from Table S-IV [48] and Table S-XIV [48] that $\mathrm{Te}_{23}\left(\mathrm{Te}_{3}\right.$ in Fig. S7 [48]), which supports linear growth on the MRCL, is the most favorable configuration.

In generating pentamers from tetramers, 108 candidates have been analyzed (see the fourth and the fifth rows of Fig. S7 [48]). These candidates are derived from seven parents; thus we examine seven families in this generation. The first significant difference in terms of the number of families between flat and curved graphene is realized in pentamers. $\mathrm{P}_{22}$ $\left(0^{\circ}\right)$ was selected for next generation but the corresponding geometry $\mathrm{P}_{33}\left(52^{\circ}\right)$ fails at the pool-selection stage. Linear hydrogen chain formation on the first and the second MRCL becomes definite with the configurations $\mathrm{P}_{42}, \mathrm{P}_{40}$, and $\mathrm{P}_{28}$. In addition, armchair-type geometry reappears with $\mathrm{P}_{31}$ and $\mathrm{P}_{37}$. It is interesting to note that, although $\mathrm{P}_{30}$ is closer to the MRCL than $\mathrm{P}_{38}, \mathrm{P}_{30}$ is eliminated. The reason can be that most of the parents on the MRCL lift carbon atoms and the formation of kink on MRCL makes it easier to adsorb a hydrogen atom. That is, the reaction barrier is lowered, giving rise to a mechanical chain reaction. The same configuration on flat graphene $\mathrm{P}_{21}$ was also transferred to the next generation. There are two families $\mathrm{D}_{2} \operatorname{Tr}_{8} \operatorname{Te}_{18} \mathrm{P}$ and $\mathrm{D}_{6} \operatorname{Tr}_{14} \mathrm{Te}_{27} \mathrm{P}$ in pentamers, which cannot produce any successful candidates. Nevertheless, we proceed them to the next generation in order to check the growth of lines across the MRCL. In tetramers, it was possible to have linear configurations making $60^{\circ}$ angles with the MRCL but in pentamers those formations are all eliminated and only lines along the MRCL are favored, a direct consequence of curvature. In $90^{\circ}$ bending, this effect becomes more pronounced, as it will be discussed below.

Finally, in generating hexamers from pentamers, 195 candidates from nine parents are investigated (see the sixth and the 

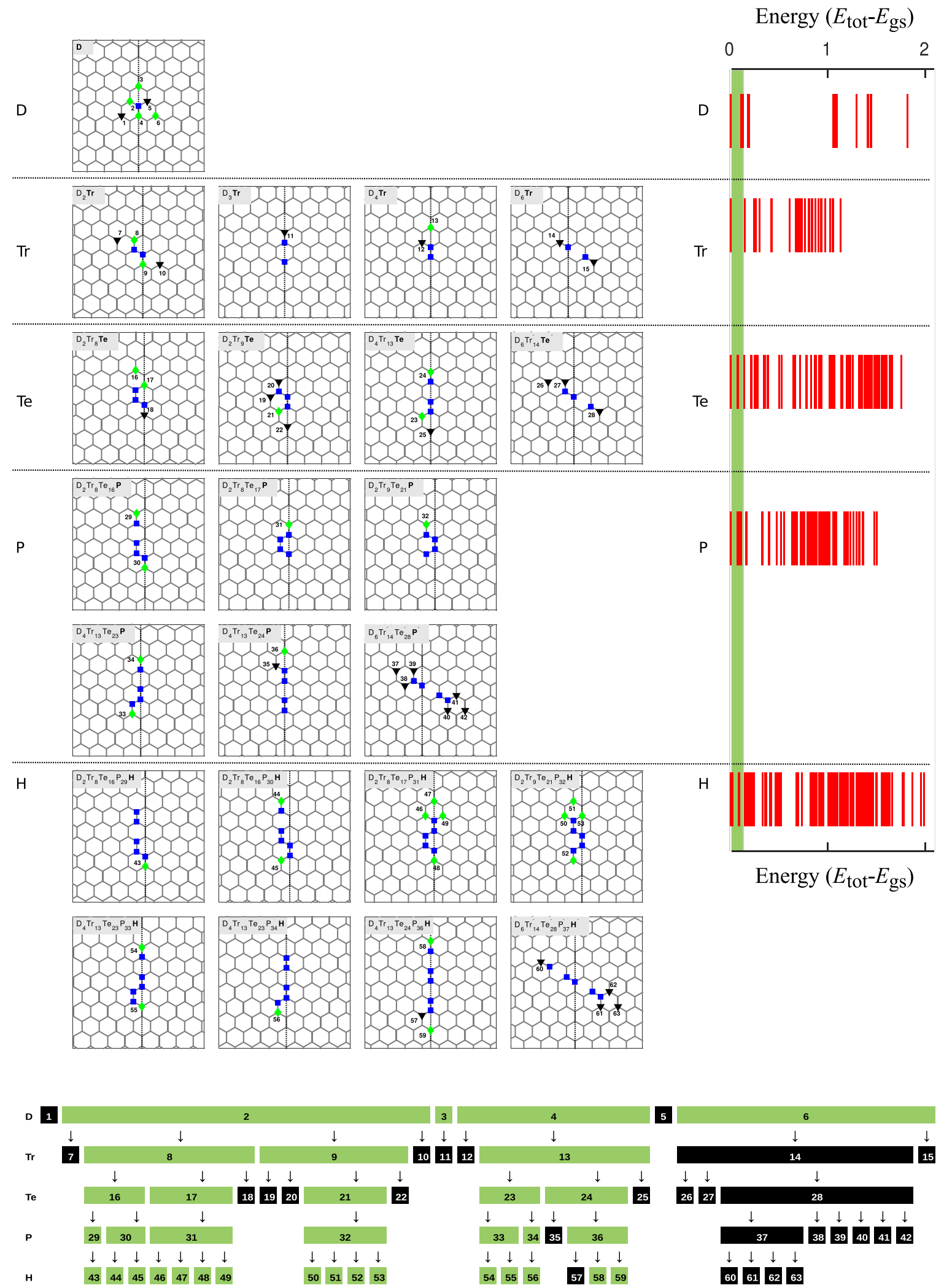

FIG. 7. Evolution of generations during the hydrogenation of curved graphene $\left(90^{\circ}\right)$. The same color codes and notation are applied as in Fig. 4. The complete list of simulated structures can be found in Fig. S8 [48].

seventh rows of Fig. S7 [48]). The second criterion of the pool selection, which is related to the symmetry properties, is not taken into account in order to display all possible preferential configurations. $\mathrm{H}_{53}, \mathrm{H}_{54}$, and $\mathrm{H}_{70}$ (Fig. 6) are eliminated in curved graphene $52^{\circ}$ bending but their corresponding configurations $\mathrm{H}_{47}$ and $\mathrm{H}_{46}$ in flat graphene are stable, which indicates that growth of single hydrogen lines in directions except the MRCL direction are suppressed. In summary, the most stable 
configurations have an armchair-type pattern $\left(\mathrm{H}_{58}\right.$ and $\left.\mathrm{H}_{64}\right)$. Both evolve from $\mathrm{D}_{2}$ but follow different paths. In addition, single-line formation is also favored. It is preferable when there are no unpaired hydrogens, while armchair-type orientation has the minimum energy even with unpaired hydrogens.

In order to examine directed growth for larger numbers of hydrogen atoms, we consider single-line and armchair-type configurations of 10 atoms. When hydrogen atoms lie along the MRCL, binding energy per hydrogen atom is enhanced by 116 $\mathrm{meV}$ and $131 \mathrm{meV}$ compared to those that lie across the MRCL with $60^{\circ}$ angle for single-line and armchair-type geometries, respectively. For $52^{\circ}$ bending, the maximum length of stable single lines is as short as a tetramer across the MRCL with an angle of $60^{\circ}$.

$90^{\circ}$ bending. Relative total energies and the binding energies for $90^{\circ}$-bent graphene are listed in Tables S-VII-S-XI and Tables S-XVII-S-XXI. In Fig. 5(c), the most stable monomer, namely the parent of the dimers marks the position of the MRCL for $90^{\circ}$-bent graphene. The family tree is shown in Fig. 7, whereas a complete list of tested configurations can be found in Fig. S8 [48].

The first difference between $52^{\circ}$ and $90^{\circ}$ bending appears in the trimer generation. $\operatorname{Tr}_{14}$ is suppressed due to the stronger curvature effect in $90^{\circ}$ bending, which succeeded in $52^{\circ}$. This means that only the lines as short as a hydrogen dimer are stable if they are not aligned with the MRCL. The family $\mathrm{D}_{6} \operatorname{Tr}\left(90^{\circ}\right)$ cannot produce any candidates that can succeed the pool selection. However, we proceed this family in order to check the energetics of lines across the MRCL. Family $\mathrm{D}_{2} \operatorname{Tr}_{8} \mathrm{Te}_{18} \mathrm{P}\left(52^{\circ}\right)$ is not observed in $90^{\circ}$ bending, because its parent was eliminated in the previous generation. Family $\mathrm{D}_{4} \operatorname{Tr}_{13} \mathrm{Te}_{23} \mathrm{P}\left(90^{\circ}\right)$ is similar to $\mathrm{D}_{4} \operatorname{Tr}_{13} \mathrm{Te}_{22} \mathrm{P}\left(52^{\circ}\right)$ except the configuration $\mathrm{P}_{38}$, which is also eliminated due to stronger curvature. The differences between $52^{\circ}$ and $90^{\circ}$ bending become more clear in hexamers. Even though $\mathrm{D}_{2} \operatorname{Tr}_{8} \mathrm{Te}_{16} \mathrm{P}_{28} \mathrm{H}\left(52^{\circ}\right)$ and $\mathrm{D}_{2} \mathrm{Tr}_{8} \mathrm{Te}_{16} \mathrm{P}_{29} \mathrm{H}\left(90^{\circ}\right)$ originate from the same parents, higher curvature does not allow succession of the configurations which are not on the MRCL. In $52^{\circ}$ bending, hydrogens can prefer to be ordered along a short line across the MRCL within the same family, whereas $90^{\circ}$ bending does not allow such a geometry. $\mathrm{H}_{52}\left(52^{\circ}\right)$, which was eliminated, appears as a successful candidate as $\mathrm{H}_{43}\left(90^{\circ}\right)$. This geometrical change reveals that single line formation on the second MRCL does not continue in $90^{\circ}$ bending. In the family $\mathrm{D}_{2} \operatorname{Tr}_{8} \operatorname{Te}_{16} \mathrm{P}_{30} \mathrm{H}$, $\mathrm{H}_{44}$ which is the same as $\mathrm{H}_{55}\left(52^{\circ}\right)$, creates a single line on the second MRCL. Both $\mathrm{H}_{58}$ and $\mathrm{H}_{59}$ form a single line on the MRCL. $\mathrm{H}_{59}$ leaves an unoccupied site for this reason; $\mathrm{H}_{59}$ is less favored in total energy when compared to $\mathrm{H}_{58} . \mathrm{H}_{57}\left(90^{\circ}\right)$ is eliminated during the pool selection. However, $\mathrm{H}_{74}\left(52^{\circ}\right)$, which is identical to $\mathrm{H}_{57}\left(90^{\circ}\right)$, succeeds through the whole selection process. This difference is originating from the the kink formation being stronger for $90^{\circ}$ bending than it is in $52^{\circ}$ bending. In summary, the main difference between $52^{\circ}$ and $90^{\circ}$ bending is in the length of hydrogen lines if they are not aligned with the MRCL. The number of eliminated candidates, especially in hexamer generation, are less than those of $52^{\circ}$ bending due to the decrease in ROC. This result indicates that families of pentamer and hexamer generations are more stable than in $52^{\circ}$ bending. As it is the case for $52^{\circ}$-bent graphene, armchair-type geometry is more favorable than the linear geometry along the MRCL. Binding energy per hydrogen atom in the armchair-type configuration is about $40 \mathrm{meV}$ more than that of linear configuration. Growth of single line and armchair-type configurations consisting of 10 hydrogen atoms are compared along and across the MRCL and it is found that the binding energies per adsorbate are enhanced by $200 \mathrm{meV}$ and $223 \mathrm{meV}$ for single line and armchair-type geometries, respectively.

Considering lines consisting of six hydrogen atoms, bending increases the binding energies by $151 \mathrm{meV}(176 \mathrm{meV})$ and $265 \mathrm{meV}(289 \mathrm{meV})$ per hydrogen atom for $52^{\circ}\left(90^{\circ}\right)$ bending with single line and armchair-type configurations, respectively. This is a clear indication that curvature favors directed growth of hydrogen lines and it may overcome substrate effects.

Conclusion. High-throughput simulations show that our evolutionary algorithm is able to predict more stable configurations than those studied before. It is more preferable for hydrogen to adsorb on lines rather than making clusters, which obey the symmetries of the hexagonal lattice. Moreover, the line formation has a preferred crystallographic orientation, namely the armchair direction, while growth along the zigzag direction is suppressed. Combined with the effect of bending on chemical reactivity, the selection process eliminates hydrogen lines which are not aligned with the MRCL of bent graphene and a directed growth becomes possible. At intermediate bending angles, line formations crossing the MRCL are possible up to tetramers. When the ROC is smaller, the length shortens down to a hydrogen dimer. The growth can be viewed as a mechanical chain reaction. The reaction barrier is lowered by both bending of the surface and proximity to an occupied site. As a result kinked graphene can be fabricated, where the electrons on the opposite sides of the hydrogen line are decoupled [29].

Acknowledgments. H.S. acknowledges support from BAGEP program of Bilim Akademisi- the Science Academy, Turkey. Part of the numerical calculations are carried out at TUBITAK ULAKBIM High Performance and Grid Computing Center.
[1] Y. Lu and Y. P. Feng, Nanoscale 3, 2444 (2011).

[2] R. Balog, B. Jorgensen, L. Nilsson, M. Andersen, E. Rienks, M. Bianchi, M. Fanetti, E. Laegsgaard, A. Baraldi, S. Lizzit, Z. Sljivancanin, F. Besenbacher, B. Hammer, G. P. Thomas, P. Hofmann, and L. Hornekaer, Nat. Mater. 9, 315 (2010).

[3] C. Lin, Y. Feng, Y. Xiao, M. Dürr, X. Huang, X. Xu, R. Zhao, E. Wang, X.-Z. Li, and Z. Hu, Nano Lett. 15, 903 (2015).

[4] D.-S. Zhang and J. Li, Phys. Lett. A 378, 68 (2014).
[5] D. Haberer, D. V. Vyalikh, S. Taioli, B. Dora, M. Farjam, J. Fink, D. Marchenko, T. Pichler, K. Ziegler, S. Simonucci, M. S. Dresselhaus, M. Knupfer, B. Büchner, and A. Grüneis, Nano Lett. 10, 3360 (2010).

[6] J. O. Sofo, A. S. Chaudhari, and G. D. Barber, Phys. Rev. B 75, 153401 (2007).

[7] P. Chandrachud, B. S. Pujari, S. Haldar, B. Sanyal, and D. G. Kanhere, J. Phys.: Condens. Matter 22, 465502 (2010). 
[8] D. C. Elias, R. R. Nair, T. M. G. Mohiuddin, S. V. Morozov, P. Blake, M. P. Halsall, A. C. Ferrari, D. W. Boukhvalov, M. I. Katsnelson, A. K. Geim, and K. S. Novoselov, Science 323, 610 (2009).

[9] B. S. Pujari, S. Gusarov, M. Brett, and A. Kovalenko, Phys. Rev. B 84, 041402 (2011).

[10] Z. F. Wang, Y. Zhang, and F. Liu, Phys. Rev. B 83, 041403 (2011).

[11] W. Bao, F. Miao, Z. Chen, H. Zhang, W. Jang, C. Dames, and C. N. Lau, Nat. Nanotechnol. 4, 562 (2009).

[12] S.-Y. Lin, S.-L. Chang, F.-L. Shyu, J.-M. Lu, and M.-F. Lin, Carbon 86, 207 (2015).

[13] L. A. Chernozatonskii, P. B. Sorokin, and J. W. Brüning, Appl. Phys. Lett. 91, 183103 (2007).

[14] L. A. Chernozatonskii and P. B. Sorokin, J. Phys. Chem. C 114, 3225 (2010).

[15] V. V. Shunaev and O. E. Glukhova, J. Phys. Chem. C 120, 4145 (2016).

[16] O. Glukhova and M. Slepchenkov, Nanoscale 4, 3335 (2012).

[17] R. Balog, B. Jørgensen, J. Wells, E. Lægsgaard, P. Hofmann, F. Besenbacher, and L. Hornekær, J. Am. Chem. Soc. 131, 8744 (2009).

[18] A. Rossi, S. Piccinin, V. Pellegrini, S. de Gironcoli, and V. Tozzini, J. Phys. Chem. C 119, 7900 (2015).

[19] S. Park, D. Srivastava, and K. Cho, Nano Lett. 3, 1273 (2003).

[20] P. Ruffieux, O. Gröning, M. Bielmann, P. Mauron, L. Schlapbach, and P. Gröning, Phys. Rev. B 66, 245416 (2002).

[21] G. Sclauzero and A. Pasquarello, Phys. Rev. B 85, 161405 (2012).

[22] T. Cavallucci and V. Tozzini, J. Phys. Chem. C 120, 7670 (2016).

[23] V. Tozzini and V. Pellegrini, J. Phys. Chem. C 115, 25523 (2011).

[24] S. Goler, C. Coletti, V. Tozzini, V. Piazza, T. Mashoff, F. Beltram, V. Pellegrini, and S. Heun, J. Phys. Chem. C 117, 11506 (2013).

[25] V. D. Camiola, R. Farchioni, T. Cavallucci, A. Rossi, V. Pellegrini, and V. Tozzini, Front. Mater. 2, 3 (2015).

[26] D. W. Boukhvalov, M. I. Katsnelson, and A. I. Lichtenstein, Phys. Rev. B 77, 035427 (2008).

[27] F. Mercuri, Int. J. Hydrogen Energy 42, 21191 (2017).

[28] T. Low, V. Perebeinos, J. Tersoff, and P. Avouris, Phys. Rev. Lett. 108, 096601 (2012).

[29] J. T. Rasmussen, T. Gunst, P. Bøggild, A.-P. Jauho, and M. Brandbyge, Beilstein J. Nanotechnol. 4, 103 (2013).

[30] H. Sevinçli and M. Brandbyge, Appl. Phys. Lett. 105, 153108 (2014).

[31] Z. Sljivancanin, M. Andersen, L. Hornekær, and B. Hammer, Phys. Rev. B 83, 205426 (2011).

[32] Z. Sljivancanin, E. Rauls, L. Hornekaer, W. Xu, F. Besenbacher, and B. Hammer, J. Chem. Phys. 131, 084706 (2009).

[33] Y. Ferro, D. Teillet-Billy, N. Rougeau, V. Sidis, S. Morisset, and A. Allouche, Phys. Rev. B 78, 085417 (2008).
[34] M. Moaied, J. A. Moreno, M. J. Caturla, F. Ynduráin, and J. J. Palacios, Phys. Rev. B 91, 155419 (2015).

[35] S. Casolo, O. M. Løvvik, R. Martinazzo, and G. F. Tantardini, J. Chem. Phys. 130, 054704 (2009).

[36] T. Fei Cao, L. Feng Huang, X. Hong Zheng, P. Lai Gong, and Z. Zeng, J. Appl. Phys. 113, 173707 (2013).

[37] N. Rougeau, D. Teillet-Billy, and V. Sidis, Chem. Phys. Lett. 431, 135 (2006).

[38] L. Hornekær, E. Rauls, W. Xu, Z. Šljivančanin, R. Otero, I. Stensgaard, E. Lægsgaard, B. Hammer, and F. Besenbacher, Phys. Rev. Lett. 97, 186102 (2006).

[39] Z. Šljivančanin, Phys. Rev. B 84, 085421 (2011).

[40] M. S. Alam, F. Muttaqien, A. Setiadi, and M. Saito, J. Phys. Soc. Jpn. 82, 044702 (2013).

[41] M. Andersen, L. Hornekær, and B. Hammer, Phys. Rev. B 86, 085405 (2012).

[42] R. Balog, M. Andersen, B. Jørgensen, Z. Sljivancanin, B. Hammer, A. Baraldi, R. Larciprete, P. Hofmann, L. Hornekær, and S. Lizzit, ACS Nano 7, 3823 (2013).

[43] B. Aradi, B. Hourahine, and T. Frauenheim, J. Phys. Chem. A 111, 5678 (2007).

[44] M. Elstner, D. Porezag, G. Jungnickel, J. Elsner, M. Haugk, T. Frauenheim, S. Suhai, and G. Seifert, Phys. Rev. B 58, 7260 (1998).

[45] P. Hohenberg and W. Kohn, Phys. Rev. 136, B864 (1964).

[46] W. Kohn and L. J. Sham, Phys. Rev. 140, A1133 (1965).

[47] H. J. Monkhorst and J. D. Pack, Phys. Rev. B 13, 5188 (1976).

[48] See Supplemental Material at http://link.aps.org/supplemental/ 10.1103/PhysRevMaterials.2.073406 for further details about high-throughput calculations on flat and curved graphene and also DFT- DFTB comparison results from dimer to hexamer configurations.

[49] L. Hornekær, Z. Šljivančanin, W. Xu, R. Otero, E. Rauls, I. Stensgaard, E. Lægsgaard, B. Hammer, and F. Besenbacher, Phys. Rev. Lett. 96, 156104 (2006).

[50] J. Kerwin and B. Jackson, J. Chem. Phys. 128, 084702 (2008).

[51] T. Zecho, A. Güttler, X. Sha, B. Jackson, and J. Küppers, J. Chem. Phys. 117, 8486 (2002).

[52] M. Li, L. Wang, N. Yu, X. Sun, T. Hou, and Y. Li, J. Mater. Chem. C 3, 3645 (2015).

[53] T. F. Cao, L. F. Huang, X. H. Zheng, W. H. Zhou, and Z. Zeng, J. Chem. Phys. 139, 194708 (2013).

[54] P. Merino, M. Švec, J. I. Martínez, P. Mutombo, C. Gonzalez, J. A. Martín-Gago, P. L. de Andres, and P. Jelinek, Langmuir 31, 233 (2014).

[55] Q. Lu, M. Arroyo, and R. Huang, J. Phys. D 42, 102002 (2009).

[56] See Supplemental Material at http://link.aps.org/supplemental/ 10.1103/PhysRevMaterials.2.073406 for further details about evolution of generations on flat graphene. 